\title{
The encoding of perceptual information in the organization of individual stimulus patterns*
}

\author{
JOSEPH S. LAPPIN, C. R. SNYDER, and CHERYL BLACKBURN \\ Vanderbilt University, Nashville, Tennessee 37203
}

Two experiments compared the effectiveness of variable physical dimensions and relationships among the components of individual stimulus patterns as means for encoding perceptual information. Four different codes were constructed in which letters (A through $P$ ) were represented by redundant combinations of the shape and brightness of the four component forms in each stimulus pattern. Three of the codes differed in terms of the physical variables that were redundant, and a fourth code was designed to simplify the relationships within individual stimulus patterns. Ss were asked to identify each pattern by naming its letter label as rapidly as possible. Differences in the speed of identification between codes and between individual patterns within codes indicated that perceptual information was effectively encoded by the organization of relationships within individual stimulus patterns. The representation of stimulation in terms of relationships among components has several implications for models of human information processing.

An important assumption underlying a large portion of research in perception and information processing is that stimulation may be represented as a set of discrete elementary components. Although the identity of these elementary components is not often specified, their existence is implicit in most theory and research concerned with an S's limited capacity for processing stimulation (e.g., Broadbent, 1958; Miller, 1956; Norman, 1969). That is, a limited capacity is usually described by the number of elements processed per unit time by various components of the processing mechanism (Estes \& Taylor, 1966; Lappin \& Ellis, 1970; Sperling, 1967; Sternberg, 1966). Major research problems have concerned the interactionsindependence, interdependence, and interference-among discrete stimulus items presented in close temporal proximity (e.g., Broadbent, 1958; Eriksen \& Lappin, 1967 ; Kristofferson, 1967; Lappin, 1967; Garner \& Morton, 1969; Garner \& Flowers, 1969).

Contrary to this representation of stimulation, Gestalt psychologists observed several years ago that some patterns seem simpler and more easily recognizable than others composed of the same physical elements. Gestalt theory held that perception was determined by the relations among stimulus elements, in contrast to the structuralist and associationist construction of a percept as a linear combination of component cues. The distinction between these two

*This research was supported in part by a grant from the Graduate Research Council of Vanderbilt University, The authors gratefully acknowledge the advice of Keith Clayton and Robert Fox on earlier versions of this paper. representations may be seen in the possibility of defining relationships (e.g., "same," "greater than") that are invariant with the particular physical elements and of describing a series or set of these relations by an inductive function (e.g., "all elements are the same," or "each element is larger than its neighbor to the left"), which is invariant with the number of elements in the pattern. This possibility has been well illustrated in the work of James Gibson (1950). Gibson has argued that the perceptually appropriate representation of stimulation should be based upon such aspects of the organization of relationships within individual stimulus arrays-upon the "higher order variables" of stimulation. Particular patterns may thus be "good" or "simple" because the interrelations among their components have a simple representation by the perceptual system.

A counterexplanation which is frequently offered for the existence of apparently simple patterns is based on functionalism: the functionally effective stimulus elements are only those variables which differentiate individual members from a larger set of alternative stimuli. Simple patterns may thus be those which occur frequently, are sampled from a small set of alternatives, or have a high degree of redundancy among their components. As Garner (1970) puts it, "Good patterns have few alternatives." Garner and his associates have provided evidence for this hypothesis by demonstrating, for example, that the number of subjective alternatives to a specific pattern is negatively correlated with ratings of the "goodness" of visual spatial patterns and positively correlated with the listening time required for accurate reproduction of auditory temporal patterns (see Garner, 1966, 1970). But, of course, this evidence has alternative interpretations. The correlation between the number of subjective alternatives to a pattern and its simplicity does not imply a particular causal relation between these variables. It is equally plausible that the existence of a small number of alternatives is a consequence, rather than a cause (as Garner seems to imply), of the geometric simplicity of the pattern.

Both "lower order" stimulus variables and "higher order" relationships have been shown to be sufficient and potent determinants of perception, but there has been no comparison of the effectiveness of these two aspects of stimulation for encoding information. The purpose of the following experiments is to provide such a comparison. These experiments are intended as demonstrational and exploratory rather than as tests of well-formed theoretical predictions.

\section{EXPERIMENT 1}

Method

Stimuli. Experiment 1 was designed to compare the effectiveness of several different methods of encoding perceptual information within a given restricted set of physical variables. The visual patterns investigated were composed of a horizontal series of four forms varying in brightness and shape. The shapes of forms were circle and square, $1 / 2$ in. in diam, separated by $1 / 4$ in. Four values of brightness were selected-white, light gray, dark gray, and black. These values were easily discriminable and spaced at approximately equal subjective intervals (as informally judged by the Es).

Codes. Four different sets of 16 stimulus patterns were constructed by varying the shape and brightness of the four component forms of the patterns. Each of these sets of patterns was used to encode or represent letters of the alphabet ( $A$ through $P$ ) by assigning a letter as a label for each pattern. The four codes are shown in Fig. 1. Each code may be considered as a sample of 16 patterns ( 4 bits) selected from a population of 256 patterns ( 8 bits) composed of all possible combinations of four component forms varying in four alternative combinations of brightness and shape. Thus, each code carried 4 bits of information and was constrained by 4 bits of redundancy. The codes differed with respect to the kind of redundancy that constrained the set of patterns. In Code D (redundant dimensions), all four components carried an equivalent amount of information, but the two 


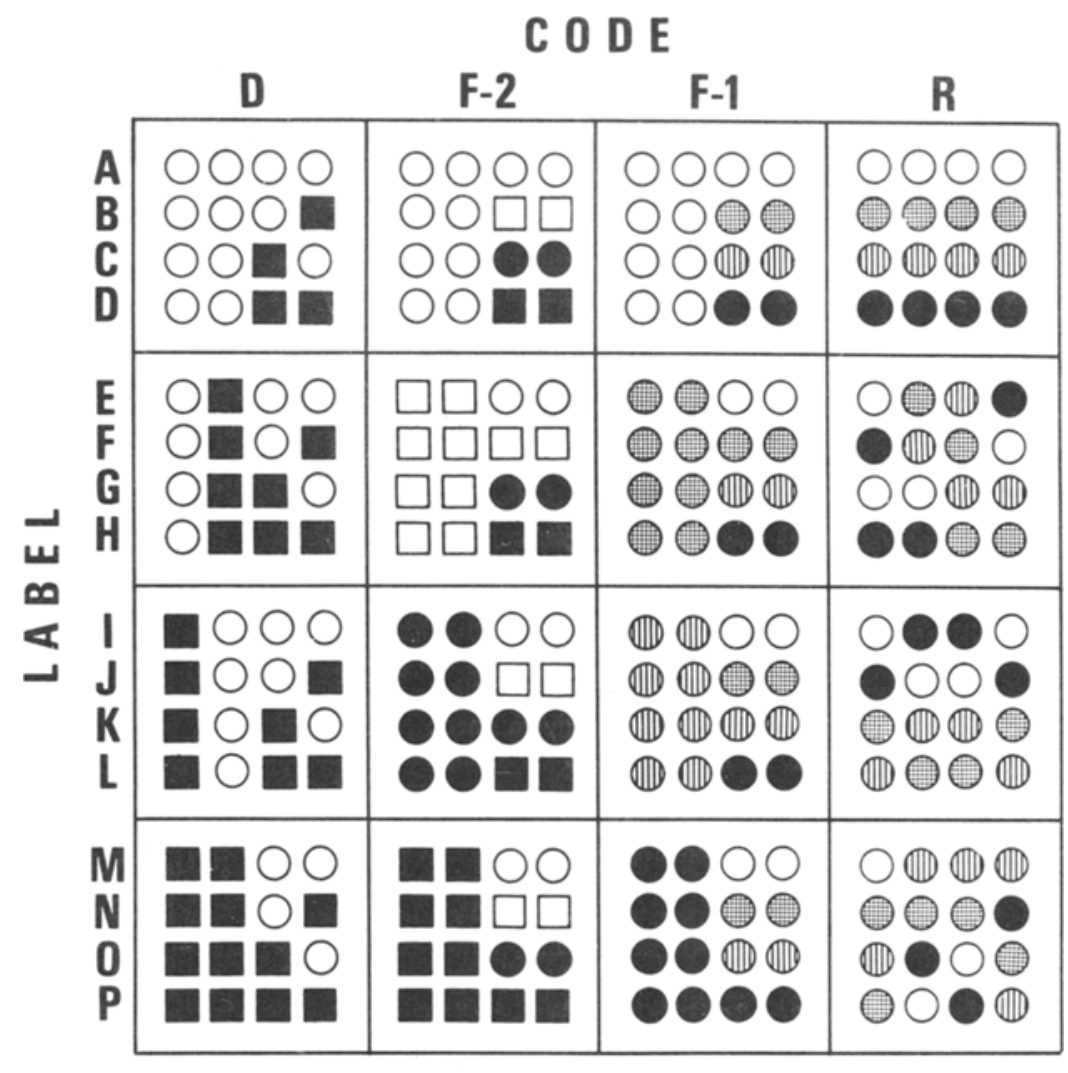

Plght gray (ID dark gray and let the left pair of forms have a weight of 4 and the right pair a weight of 1 ; then the weighted sum of the two brightness values generates the integers 0 to 15 . The same system may be used to recognize the patterns in Codes $\mathrm{D}$ and F-2. Code $R$, however, has a more complex structure when given this representation as a linear combination of physical variables. A principal components analysis of the structure among the four variable forms (representing brightnesses with four consecutive integers) yielded orthogonal components that accounted for $37.6 \%, 29.1 \%, 25.9 \%$, and $7.4 \%$ of the total variance. Four component variables are necessary to completely discriminate among the 16 patterns of Code $R$, although three variables are sufficient to distinguish among about 15 of the 16 patterns. The comparison between Code $R$ and the other codes may thus be considered a test of the appropriateness of the representation of these visual patterns as linear combinations of independent physical variables. Because Code $R$ is relatively complex under this representation, superior performance with Code $R$ would indicate that the perceptually effective structure of these patterns is not captured by this representation.

An alternative representation may be based upon the pairwise relations among the four component forms. With this relational representation we need assume only that the $S$ obtains comparative rather than absolute information about the brightnesses of the components. Let $a, b, c$, and $d$ represent the four forms from left to right, respectively. For the one-dimensional codes, F-1 and R, we may define two binary relations based on the ordered set of four brightnesses: =, which is reflexive, symmetric, and transitive; and <, which is transitive. (Additional numerical properties of these relations are not applicable here.) For the two-valued dimensions of Codes $D$ and $\mathrm{F}-2$, the ordinal relation $<$ needs to be only a nonequivalence, $\neq$. For Codes $R, F-1$, and $D$, three such binary relations are necessary and sufficient to describe the organization of the pattern; but for Code F-2, six relations are appropriate, since there are two dimensions of variation. These binary relations define a quasiseries of the four forms on each dimension. Six classes of organization for the patterns in Codes $D, F-1$, and $R$ may be distinguished as follows. The classes are ranked on the basis of the homogeneity of the series of relations among adjacent pairs of forms, with greater simplicity given to the equivalence relation. 
Class 1: $[a=b=c=d]$

Class 2: $[\mathrm{a}<\mathrm{b}<\mathrm{c}<\mathrm{d}]$

$$
\text { OR }[a>b>c>d]
$$

Class 3: $(a=b, c=d, b \neq c]$

$$
\text { OR }[a=d, b=c, a \neq b]
$$

Class 4: $[a=b=c, c \neq d]$

$$
\mathrm{OR}[\mathrm{a} \neq \mathrm{b}, \mathrm{b}=\mathrm{c}=\mathrm{d}]
$$

Class 5: $[a=c=d, a \neq b]$

$$
\text { OR }[a=b=d, b \neq c]
$$

Class 6: $[\mathrm{a}<\mathrm{b}>\mathrm{c}<\mathrm{d}]$

$$
\text { OR }[a>b<c>d]
$$

The average ranks of the patterns in Codes $D, F-1$, and $R$ are $3.5,2.5$, and 2.75, respectively. Although Code F-1 has the least average complexity by this measure, it also has the least variability in the organization of its patterns. Insofar as the varying organizations of the patterns in a code serve as cues to discriminate among the patterns, this lack of differentiation should render the code less effective. Code $R$, it may be noted, lists the patterns in order of increasing organizational complexity; this seems likely to facilitate the usefulness of the organization as a discriminative cue.

The patterns in Code F-2 may be ranked in three classes, in which the organization $a=b=c=d$ holds for both, for one, or for neither of the two dimensions, color and shape. Code F-2 can probably reasonably be considered as more complex than the other three codes, by virtue of its having two dimensions of relationship among the component forms, but a more quantitative comparison with the one-dimensional codes would not be. meaningful.

The general point is that the predictions one should make about the relative effectiveness of these codes depend upon one's assumptions about the perceptually relevant properties of the stimulus patterns.

Tasks and procedures. The effectiveness of the four codes was

\begin{tabular}{|c|c|c|c|c|}
\hline & \multicolumn{4}{|c|}{ Code } \\
\hline & $\mathbf{R}$ & D & $F-1$ & $\mathbf{F}-2$ \\
\hline Day 1 & 92.0 & 99.0 & 85.7 & 118.3 \\
\hline Day 2 & 54.7 & 75.7 & 75.2 & 91.7 \\
\hline Day 3 & 55.3 & 59.6 & 61.6 & 71.2 \\
\hline Day 4 & 42.5 & 44.8 & 51.4 & 59.6 \\
\hline
\end{tabular}
measured by the speed of identifying

Table 1

Average Card-Sort Time (Seconds) for Each Code on Each Day in Experiment 1 individual patterns within each code list. The assumption is that this measure reflects the speed with which the stimulus information is decoded by the perceptual system-rather than, say, the associative strength of the pattern-letter pairs. A few minutes of study and practice were sufficient to eliminate errors in identifying the patterns in each of the code lists, and we wished to determine if persistent differences in the effectiveness of these codes might be obtained after extended familiarity and training. The RTs for identifying the patterns served this purpose quite well.

There were two separate tasks in which the $\mathbf{S}$ was asked to produce the letter label for each pattern as rapidly as possible. In the RT task, each pattern was presented individually in the tachistoscope. When he was ready, the $\mathbf{S}$ depressed a telegraph key, which presented the stimulus after a $0.5-\mathrm{sec}$ delay. The $S$ released the key simultaneously with his verbal response of naming the letter. The timer was stopped, and the stimulus terminated when the key was released. The RT task consisted of 32 presentations, each pattern presented twice. The order of presentation was randomized for each set of 16 trials.

In a separate card-sorting task, the $S$ was asked to sort as rapidly as possible through a shuffled pack of $483 \times 5$ in. cards, in which each of the possible patterns occurred three times. The $S$ named the letter for the pattern on each card, and the total time required to sort through the 48 cards was recorded.

Both RT and card-sort tasks were performed in each experimental session in the following order: $32 \mathrm{RT}$ trials, 2 card sorts, 32 RTs, 2 card sorts, 32 RTs, and 2 card sorts. A session required about $45 \mathrm{~min}$. S used each code list in four separate sessions: two consecutive sessions in the first half of the experiment and two more in the second half. There were 16 sessions, with 2 successive days per code, and 1 day between different codes. A master list of the correct letter-pattern associations was present for the entire session on the first day with each code (Sessions 1, 3, 5, 7) and absent on the second day with the code. For the third day on each code (Sessions 9, 11, 13, and 15), the master list was presented only for a 3-min study time at the beginning of the session and was absent on the fourth and last day for the code. The order of presentation of the codes was counterbalanced within and between Ss.

Subjects. Four female undergraduates served as $S s$ in the experiment. Participation was voluntary and paid.

Apparatus. An Iconix three-channel tachistoscope was used for the RT task. The adaptation field was illuminated at $40 \mathrm{fL}$, and the stimulus field was illuminated at $52 \mathrm{fL}$. The vertical visual angle of the stimulus was $40 \mathrm{~min}$; the horizontal visual angle was 4 deg.

\section{Results and Discussion}

Card sorts. Table 1 gives the average total time required to sort the 48 cards on each of the 4 days under each code. In subsequent analyses, data from the first session in each condition are not included because of the understandably large practice effects which differed between codes in the first session, and because the Ss always had the master list to refer to in the first session. The average times for card sorts in the last three sessions of each condition were as follows: $R$, $50.8 \mathrm{sec} ; \mathrm{D}, 60.0 \mathrm{sec} ; \mathrm{F}-1,62.8 \mathrm{sec}$; $\mathrm{F}-2,74.2 \mathrm{sec}$. By analysis of variance ( 4 codes by 3 days by 6 trials by $4 \mathrm{Ss}$ ), the four code lists were significantly different, $F(3,9)=8.06, p<.01$. By a Newman-Keuls post hoc comparison, Code F-2 was slower than D and F-1 at $\mathrm{p}<.05$ and slower than $\mathrm{R}$ at $\mathrm{p}<.01$. Significant main effects were also obtained for days, $F(2,6)=18.09$, $\mathrm{p}<.01$, for trials within days, $F(5,15)=8.10, p<.001$, and for $\mathrm{Ss}$, $F(3,90)=481.6, p<.001$. Most of the $S$ variance was contributed by one $S$ whose times were noticeably slower and more variable than those of other Ss. None of the interactions was significant.

Reaction times. Table 2 shows that the overall effects for RTs were similar to those for card sorts. The RTs provide additional information about specific patterns for each code. Table 3 gives the average RT for identification of each pattern for each code; differences were obtained between the code lists and also

Table 2

Average RT (Seconds) for Each Code on Each Day in Experiment 1

\begin{tabular}{lllll}
\hline & \multicolumn{5}{c}{ Code } \\
\cline { 2 - 5 } & $\mathrm{R}$ & $\mathrm{D}$ & $\mathrm{F}-1$ & F-2 \\
\hline Day 1 & 2.08 & 2.26 & 1.87 & 2.39 \\
Day 2 & 1.57 & 1.79 & 1.77 & 2.06 \\
Day 3 & 1.37 & 1.53 & 1.45 & 1.64 \\
Day 4 & 1.06 & 1.21 & 1.26 & 1.45 \\
\hline
\end{tabular}


Table 3

Average RT (Seconds) for Each Pattern Under Each Code in Experiment 1

\begin{tabular}{lrrrr}
\hline Letter & \multicolumn{3}{c}{ Code } & F-1 \\
\cline { 2 - 4 } Designation & R & D & .71 & F-2 \\
\hline A & .70 & .76 & 1.25 & 1.56 \\
B & .98 & 1.38 & 1.30 & 1.67 \\
D & 1.02 & 1.63 & 1.18 & 2.01 \\
E & .88 & 1.40 & 1.68 & 1.81 \\
F & 1.18 & 1.63 & 1.24 & 1.20 \\
G & 1.34 & 1.48 & 1.58 & 2.39 \\
H & 1.11 & 1.05 & 2.12 & 2.00 \\
I & 1.17 & 1.93 & 1.90 & 1.86 \\
J & 1.15 & 1.59 & 2.11 & 2.31 \\
K & 1.23 & 1.15 & 1.35 & 1.36 \\
L & 1.37 & 1.70 & 1.89 & 1.89 \\
M & 1.16 & 2.71 & 1.38 & 2.12 \\
N & 1.37 & 1.84 & 1.75 & 1.97 \\
O & 1.51 & 1.74 & 1.47 & .90 \\
P & 1.56 & 1.34 & 1.35 & 1 \\
\hline
\end{tabular}

between the patterns within each list. By analysis of variance ( 4 codes by 16 positions within the list by 3 days by 4 Ss), the main effect for codes was significant, $E(3,9)=5.06, \mathrm{p}<.05$. By Newman-Keuls post hoc comparisons, Code $\mathbf{R}$ was faster than $\mathrm{F}-2$ at $\mathrm{p}<.05$. Positions within code lists were significantly different, $F(15,45)=4.76, p<.001$, and the effect of positions interacted with codes, $\quad F(45,135)=3.61, \quad p<.001$. The effect of positions seems to be reduced and different for Code List $R$. Significant effects were also obtained for: days, $F(2,6)=6.78, p<.05$; Ss, $F(3,270)=184.4, p<.001 ;$ and the interaction of Days by Positions, $\mathrm{F}(30,90)=2.60, \mathrm{p}<.001$.

The superiority of Code $R$ implies that the perceptual processing of stimulus information in these tasks was not based on a linear combination of evidence from independent physical variables. What, then, might be the nature of the underlying process? We wish to suggest that it is based, in part, on an analysis of relations among the component forms within individual stimulus patterns. Evidence on this point may be obtained from the RTs for identifying individual stimulus patterns given in Table 3 . As may be seen, there was considerable variability among the RTs to the patterns within each code list, and the simpler patterns seem to have been identified more rapidly. This is shown in more detail by correlations between $\mathrm{RT}$ and the class of the relational organization of each pattern. Spearman rank-order correlations between the average RT and the organizational simplicity of each pattern within each code were: $\mathrm{R}, \mathrm{r}_{\mathrm{s}}=.84, \mathrm{D}, \mathrm{r}_{\mathrm{s}}=.63 ; \mathrm{F}-1, \mathrm{r}_{\mathrm{s}}=.53 ;$ F-2, $r_{s}=.92$. With $\mathrm{df}=14$, the correlation for Code F-1 is significant at $\mathrm{p}<.05$; the other correlations are significant at $p<.01$.

The results of this experiment are not compatible with the interpretation
EXPERIMENT 2

Experiment 2 was designed to add evidence on two points. First, it replicates the main finding of Experiment 1 by again comparing Codes $R$ and F-1. Second, Experiment 2 provides information about the effects of the arrangement of items on a code list. One property that differentiates the code lists of Experiment 2 is the more obvious serial listing in Codes D, F-1, and F-2, than in Code $R$. It seems conceivable that this extra cue interferes with a perceptual association between pattern and label in Codes $D, F-1$, and F-2. Code $\mathrm{R}$ might have benefited from a lack of such interference. Experiment 2 was designed to check this possibility.

\section{Method}

Conditions. Three codes were used. the speed of identification wa dependent upon a learned associative strength between a pattern and its label. First, although performance continued to improve over the 4 days of practice with a code, there was no interaction between the amount of practice over the last 3 days and the differences among the codes [card sorts, $F(6,18)=1.27, \quad \mathrm{RTs}$, $F(6,18)=1.88$ ]. Contrary to common assumptions about the role of the number of subjective alternatives or associates to a pattern (e.g., Garner, 1966,1970 ), reliable differences were obtained between patterns which had the same number of objectively defined alternatives and among code lists with the same number of items. Moreover, preexperimental associations would not seem to favor Code R, since the initial speed of identification was slowest for Code $R$. The average card-sorting times on the first trial were: $D, 115$ sec; F-2, 124 sec; F-1, $101 \mathrm{sec}$; and $R, 132$ sec. The average RTs on the first trial were: $D, 2.78 \mathrm{sec} ; \mathrm{F}-2,2.42 \mathrm{sec}$; F-1, $2.34 \mathrm{sec}$; and $R, 3.02 \mathrm{sec}$.

The variations in $\mathrm{RT}$ both within and between code lists indicate that the speed of identification depended upon the organization of individual stimulus patterns. Several other factors, however, were potentially important in determining performance. The organization of relationships alone would obviously be inadequate to distinguish among all 16 patterns-e.g.; : in Code F-1, 4 patterns had a "Class 1 " organization and 12 had a "Class 3" organization. The mapping of the items on a code list onto the set of possible pattern organizations is probably an important determinant of performance, but we have no information about the optimal number of different organizations and how they should be arranged on the code list.
$F-1$ and $R$ were the same codes used in Experiment 1. A third code, F-1', was composed of the same patterns in Code F-1, but the patterns were randomly permuted with respect to their letter designation. The patterns labeled $\mathbf{A}$ through $\mathrm{P}$, respectively, in Code F-1 were labeled as follows in List F-1': P, E, F, M, H, A, N, K, L, O, C, D, J, I, B, G. The master list used for study listed the patterns in alphabetical order by their labels.

Tasks and procedure. There were nine experimental sessions, with 3 successive days per code and no days between codes. Ss learned a code for 3 successive days, then transferred to another code for 3 days, and a third code for the final 3 days. The order of presentation of the codes was counterbalanced across Ss. The master list was present on the first day (Sessions 1, 4, 7) for the entire session, and absent on the second and third days. All other aspects of the method were identical to Experiment 1.

Subjects. Three female undergraduates served as $S s$ in the experiment.

\section{Results and Discussion}

Card sorts. Table 4 gives the average time required to sort 48 cards under each code. By the same rationale as in Experiment 1, data from the first session were not included in the analyses. The average times for card sorts in the last two sessions were: $R$, 72.2 sec; F-1, 92.9 sec; F-1', 111.3 sec. By analysis of variance ( 3 codes by 2 days by 6 trials by $3 \mathrm{Ss}$ ), the three code lists were significantly different, $\mathrm{F}(2,24)=8.74, \quad \mathrm{p}<.05$. Newman-Keuls post hoc comparisons showed Code F-1 and F-1 to be slower than Code $R$ at $p<.05$. Significant main effects were obtained for days, $F(1,2)=59.94, p<.01$, and for trials, $F(5,10)=14.06, p<.001$. Days and trials were the only variables 
Table 4

Average Card Sort Time (Seconds) for Each Code on Each Day in Experiment 2

\begin{tabular}{lrrr} 
& \multicolumn{3}{c}{ Code } \\
\cline { 2 - 4 } & $\mathrm{R}$ & $\mathrm{F}-1$ & $\mathrm{~F}-1^{\prime}$ \\
\hline Day 1 & 90.0 & 105.0 & 142.7 \\
Day 2 & 69.0 & 99.0 & 113.3 \\
Day 3 & 53.7 & 74.7 & 77.3 \\
\hline
\end{tabular}

to interact significantly, $F(5,10)=14.56, p<.001$

Reaction times. Table 5 gives overall results for the $R T s$, which were similar to those for card sorts. Th e codes were significantly different by analysis of variance ( 3 codes by 16 positions by 2 days by $3 \mathrm{Ss}), F(2,4)=45.93, p<.01$. Further analysis by Newman-Keuls post hoc comparisons showed Code $F-1^{\prime}$ to be significantly slower than Codes $R$ and F-1 at $p<.001$. The effects of positions interacted with the codes, $F(30,60)=3.82, p<.001$, and with days, $F(30,60)=1.82, p<.05$.

Codes R, F-1, and F-1 share patterns in common, and an additional analysis of variance ( 6 patterns by 3 codes by 3 Ss) was used to determine whether the three codes differed in RTs to the common patterns. The main effect of codes was significant, $F(2,4)=18.68, p<.01$, as was the main effect of patterns, $F(5,10)=25.15, p<.001$. Codes also interacted significantly with patterns, $F(10,20)=2.78, p<.05$. Thus, there was a context effect from the entire code list which influenced the RTs to a particular pattern.

Additional information about this distinction between the contributions of the individual patterns and of the organization of the code list may be obtained from a comparison of Codes F-1 and F-1'. 'Product-moment correlations of the RTs to individual patterns in the two lists were calculated by matching the lists first with respect to letter names (or positions on the list) and secondly with respect to the patterns themselves. The correlation by letter names was +.448 ; the correlation by patterns was +.790 . The latter value is significantly higher, $t(2,13)=2.13$, $p<.05$. (Since these were only two of 16 ! permutations of the pattern-letter pairings, the absolute values of these correlations are not meaningful.) This finding adds further support to the conclision that the speed of identification of a pattern is dependent upon the properties of the individual pattern and not merely the set of alternatives within which it appears.

Additionally, an obvious serial listing of the patterns in a set appears to facilitate rather than interfere with the association between a pattern and its label. The inferiority of F-1' was manifest more at the beginning of training and diminished with practice. An unanswered question is whether or not other differences among the codes might disappear with greatly extended practice. (In retrospect, it would seem interesting to have also altered the ordering of Code $R$, which has a weaker ordering than Code $\mathrm{F}-1$, or to have given a more organizationally based ordering to Code F-1'.)

\section{GENERAL DISCUSSION}

These experiments indicate that the rate at which humans recognize patterns is dependent upon relationships among separate components of the individual stimulus pattern. This demonstration has three important implications concerning: (1) the encoding of sensory information in designing communication systems, (2) theories about pattern recognition in biological systems, and (3) the design and interpretation of research on perceptual information processing.

Consider the following problem of optimally coding sensory information: Suppose that one wished to find a one-to-one mapping of a set of symbols (e.g., the 26 letters of the English alphabet) onto a subset of physical stimuli sampled from a population of all combinations of some set of independent physical variables so as to maximize the rate and accuracy with which sequences of the encoded symbols could be received by a human operator. The present results suggest that an optimal set of physical stimuli would maximize the organizational simplicity of the relations among components within individual stimulus patterns. This general suggestion must be tempered, of course, by the limited sets of stimulus patterns tested in these experiments. One must also recognize the vagueness in the recommendation to maximize the "organizational simplicity" of individual patterns. Nevertheless, this recommendation is distinctly different from the implicit thrust of contemporary work on perceptual information processing.

The proposition that organizational aspects of stimulus patterns are critical determinants of their perceptual processing raises a major question concerning whether or not and how the necessary relational analysis might be implemented in biological systems. The answer is still quite uncertain. Results in sensory physiology do, however, lend credence to this speculation. The opposition of excitatory and inhibitory synaptic connections provides a mechanism for contrasting the input to one set of receptors with that of another, thereby amplifying differences and
Table 5

Average RT (Seconds) for Each Code on Each Day in Experiment 2

\begin{tabular}{lccc} 
& \multicolumn{3}{c}{ Code } \\
\cline { 2 - 4 } & $\mathbf{R}$ & F-1 & F-1' \\
\hline Day 1 & 2.16 & 2.09 & 3.31 \\
Day 2 & 1.78 & 2.12 & 2.65 \\
Day 3 & 1.30 & 1.60 & 1.83 \\
\hline
\end{tabular}

ordinal relations. This appears to be a widely used means of sensory coding-e.g., Ratliff (1965), von Békésy (1967), DeValois (1965), and Pfaffman (1959). James Gibson (1966) has argued that the perceptual system must be capable of extracting the relationships which are invariant in an array of sensory stimulation originating from three-dimensional objects moving in space and time. The contribution of the present experiments is to demonstrate that "higher order" relationships may be more effective than "lower order" physical variables in encoding perceptual information.

Research on perception has often assumed that a "stimulus" is a set of discrete component elements (Estes \& Taylor, 1967). The present experiments demonstrate the appropriateness of an alternative, or at least supplementary, representation, in which the "stimulus" is a function or rule that generates a series of elements from an initial element and its relations with other elements. The primary distinction between the two alternative representations is based on the use of relationships in the second alternative. A series of relationships (e.g., equality, greater than) may be defined that is invariant over many different sets of physical elements. In addition, these relations may be recursively applied, say in a left-to-right series, to generate inductively an infinite series of elements. Thus, we might suggest that the effective stimulus for perception is the organized series of relationships rather than the set of elements themselves. This suggestion echoes the writing of James Gibson and parallels recent developments in serial pattern learning (Restle, 1970).

The results of these experiments are discrepant with what one might have expected from previous identification experiments providing analyses of confusion errors (see Garner, 1962). The discrepancy is due partly to the use of RTs to a well-learned set of easily discriminable patterns, rather than the more traditional measures of confusion among stimulus items. Errors of discrimination apparently play little or no part in the perceptual processes used in the present tasks. Another reason for the apparent discrepancy with other identification 
experiments may simply be that this experiment studied the effects of stimulus properties that do not exist under the representation of stimulation as a set of discrete component elements. Stimulus sets are usually constructed from orthogonal variables in several physical dimensions. Similarly, data analyses have usually been designed to reveal effects from the covariation of variables within stimulus sets rather than from differences between individual stimulus patterns.

The results of these experiments are compatible in several ways with studies of the structure of temporal auditory patterns by Garner and his associates (e.g., Royer \& Garner, 1966; Garner \& Gottwald, 1968). They too have found large differences in the perceptual difficulty of patterns sampled from the same set of objective alternatives. Their Ss most accurately reproduced serial patterns with simple substructures composed of runs of the same element and alternations between two different elements. Garner has explained these results in terms of the number of subjective alternatives for various patterns. Such an explanation could also be given for the results of this experiment. But we wish to suggest the parsimony and heuristic value of an alternative explanation in terms of the internal organization of the individual stimulus pattern. Postulation of a set of subjective alternatives for any given pattern seems unnecessary. The representation of stimulus patterns by an organized set of internal relations also implies the operation of different processes underlying the recognition of patterns.

In a very general sense, these experiments provide more support for Gestalt representations than for structuralist and functionalist representations of stimulation. In Köhler's words, "The fact that organization depends upon relations among the local stimuli makes it entirely clear that sensory organization cannot be understood in terms of local processes as such. Independent local facts are entirely indifferent to any formal relations which may obtain between them [Köhler, 1947, p. 98]."

\section{REFERENCES}

BÉKÉSY, G. v. Sensory inhbition Princeton: Princeton University Press, 1967.

BROADBENT, D. E. Perception and communication. Oxford: Pergamon Press, 1958.

DeVALOIS, R. L. Behavioral and electrophysiological studies of primate vision. In W. D. Neff (Ed.), Contribution to sensory physiology. Vol. 1. New York: Academic Press, 1965. Pp. 137-178.

ERIKSEN, C. W., \& LAPPIN, J. S. Independence in the perception of simultaneously presented forms at brief durations. Journal of Experimental Psy chology, 1967, 73, 468-472.

ESTES, W. K., \& TAYLOR, H. A. Visual detection in relation to display size and redundancy of critical elements. Perception \& Psychophysics, 1966, 1 . 9-16.

GARNER, W. R. Uncertainty and structure as psychological concepts. NeW York: Wiley, 1962.

GARNER, W. R. To perceive is to know. American Psychologist, 1966, 21, 11-19.

GARNER, W. R. Good patterns have few alternatives. American. Scientist, 1970 $58,34-42$.

GARNER, W. R., \& FLOWERS, J, H. The effect of redundant stimulus elements on visual discrimination as a function of element heterogeneity, equal discriminability, and position uncertainty. Perception \& Psychophysics, 1969, 6, 216-220。

GARNER, W. R., \& GOTTWALD, R. L. The perception and learning of temporal patterns. Quarterly Journal of Experimental Psychology, 1968, 20, 97-109.

GARNER, W. R., \& MORTON, J. Perceptual independence: Definitions, models and experimental paradigms. Psychological Bulletin, 1969, 72, 233-259.

GIBSON, J. J. Perception of the visual world. Boston: Ho ughton Mifflin, 1950.

GIBSON, J. J. The senses considered as perceptual systems. Boston: Houghton Mifflin, 1966.

KÖHLER, W. Gestalt psychology. New York: Liveright, 1947.

KRISTOFFERSON, A. B. Attention and psychophysical time. In A. F. Sanders (Ed.), Attention and performance. Amsterdam: North-Holland, 1967.

LAPPIN, J. S. Attention in the identification of stimuli in complex visual displays. Journal of Experimental Psychology, 1968, 75, 321-328.

LAPPIN, J. S., \& ELLIS, S. H. The span of apprehension: Form identification as a function of amount of information displayed. Perception \& Psychophysics; $1970,7,65-72$.

MILLER, G. A. The magical number seven, plus or minus two: Some limits on our capacity for processing information. Psychological Review, 1956, 63, 81-97.

NORMAN, D. Memory and attention. New York: Wiley, 1969.

PFAFFMAN, $C$. The afferent code for sensory quality. The American Psychologist, 1959, 14, 226-232.

RATLIFF, F. Mach bands: Quantitative studies on neural networks in the retina. San Francisco: Holden-Day, 1965.

RESTLE, F. Theory of serial pattem learning: Structural trees. Psychological Review, 1970, 77, 481-495.

ROYER, F. L., \& GARNER, W. R. Response uncertainty and perceptual difficulty of auditory temporal patterns. Perception \& Psychophysics, 1966, 1, 41-47.

SPERLING, G. Successive approximations to a model for short-term memory. In $\mathbf{A}$. F. Sanders (Ed.), Attention and performance. Amsterdam: North-Holland, 1967.

STERNBERG, S. High-speed scanning in human memory. Science, 1966, 153, 652-654.

(Accepted for publication December 30 , 1970.) 